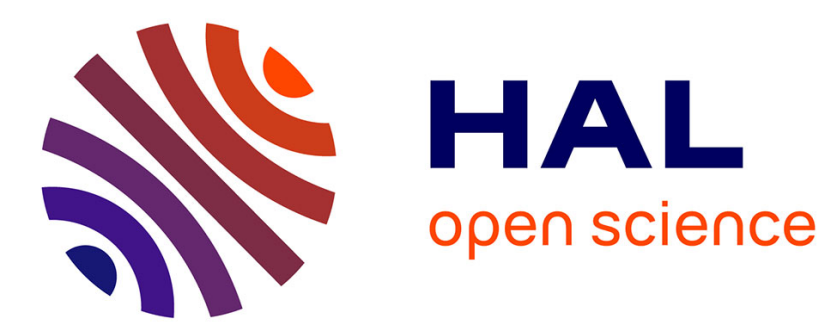

\title{
METASTABLE ORDERED COMPOUNDS FORMED DURING EPITAXIAL GROWTH
}

\author{
C. Flynn
}

\section{To cite this version:}

C. Flynn. METASTABLE ORDERED COMPOUNDS FORMED DURING EPITAXIAL GROWTH. Journal de Physique Colloques, 1988, 49 (C5), pp.C5-719-C5-730. 10.1051/jphyscol:1988594 . jpa00228091

\section{HAL Id: jpa-00228091 https://hal.science/jpa-00228091}

Submitted on 1 Jan 1988

HAL is a multi-disciplinary open access archive for the deposit and dissemination of scientific research documents, whether they are published or not. The documents may come from teaching and research institutions in France or abroad, or from public or private research centers.
L'archive ouverte pluridisciplinaire HAL, est destinée au dépôt et à la diffusion de documents scientifiques de niveau recherche, publiés ou non, émanant des établissements d'enseignement et de recherche français ou étrangers, des laboratoires publics ou privés. 
METASTABLE ORDERED COMPOUNDS FORMED DURING EPITAXIAL GROWTH

\author{
C.P. FLYNN
}

Physics Department and Materials Research Laboratory, University of Illinois at Urbana-Champaign, $1110 \mathrm{~W}$. Green street, Urbana, IL 61801. U.S.A.

\title{
Abstract
}

New ordered compounds have recently been observed to form pseudomorphically during epitaxial growth. These phases do not appear on the equilibrium bulk phase diagram. The paper first reviews the current knowledge of epitaxial strain layers. A linear stability analysis is presented of the competition among disordered phases strained by an epitaxial constraint and of the corresponding ordered epitaxial compounds. The case of volume misfit for mixed alkali halides is carried through in detail to reveal the delicate energy balance, the orientation dependences involved, and the effects of lattice anharmonicity on phase competition at the growth temperature.

\section{Introduction}

The phenomenon described here is a type of pseudomorphism in which metastable ordered compounds form during epitaxial growth. These phases do not occur in bulk equilibrium. Various pseudomorphic behaviors have been studied over the years but it seems fair to say that none is well understood. This is certainly true of the processes of interest here.

In surface science, pseudomorphism most commonly occurs when an adsorbed monolayer adopts the atomic arrangement and spacing of the substrate crystal. Usually, the growth of further monolayers causes the film to transform to the structure of lowest free energy. In any case the lattice spacing is gradually relaxed to its equilibrium value by defect mechanisms. For several decades examples have been recognized in which structures a number of monolayers thick can be grown in pseudomorphic form. These includel rocksalt alkali halides in the CsC1 structure, fcc metals grown in bcc lattices etc.; examples such as fcc Fe on $\mathrm{Cu}$ and bcc Co on GaAs have been of recent interest in connection with magnetic thin film devices. 2 Most spectacular are the cases of carbon and tin. Both grow microns thick in their metastable diamond structures in the temperature range above $\sim 300 \mathrm{~K} .3$

Over the past two years several research groups have observed epitaxial films that grow as ordered compounds in diamond and zincblende-related structures when no such ordered phases exist on the bulk phase diagram. The early results include a rhombohedral phase of $\mathrm{Si}_{1-\mathrm{x}} \mathrm{Ge}_{x}, 4$ tetragonal (CuAu) $\mathrm{Ga}_{x} \mathrm{Al}_{1-\mathrm{x}^{\mathrm{As}}}{ }^{5}$ and $\mathrm{GaAs}_{\mathrm{x}} \mathrm{Sb}_{1-\mathrm{x}^{6}}$, and chalcopyrite $\mathrm{GaAs}_{\mathrm{x}} \mathrm{Sb}_{1-\mathrm{x}},{ }^{6}$ with detailed dependences on growth orientation, temperature and kinetics that appear quite idiosyncratic.7 Figure 1 employs the example of $\mathrm{GaAs}_{x} \mathrm{Sb}_{x}$ grown on [100]InP with a diffraction pattern showing $\left[\begin{array}{lll}1 & 1 / 2 & 0\end{array}\right]$ (chalcopyrite) and [100] (CuAu) superlattice spots not present in the disordered solution. Phase separation into dilute GaAs and GaSb occurs instead in the bulk. Whether or not similar phenomena occur widely for other types of lattice is not yet clear. However, one of the earliest observations was the persistence 8 of long range order in epitaxial AgAu, which forms a continuous solid solution in the bulk, so the effect cannot be confined solely to tetrahedral coordination. All such phenomena are of general interest in the contemporary focus on the growth and understanding of new materials. A special feature of ordered compound formation, in addition, is the opportunity it brings to reduce the carrier scattering that occurs in the alternative disordered alloys that appear on the equilibrium bulk phase diagram. The possible advantages for device purposes have attracted technical interest to the area. 

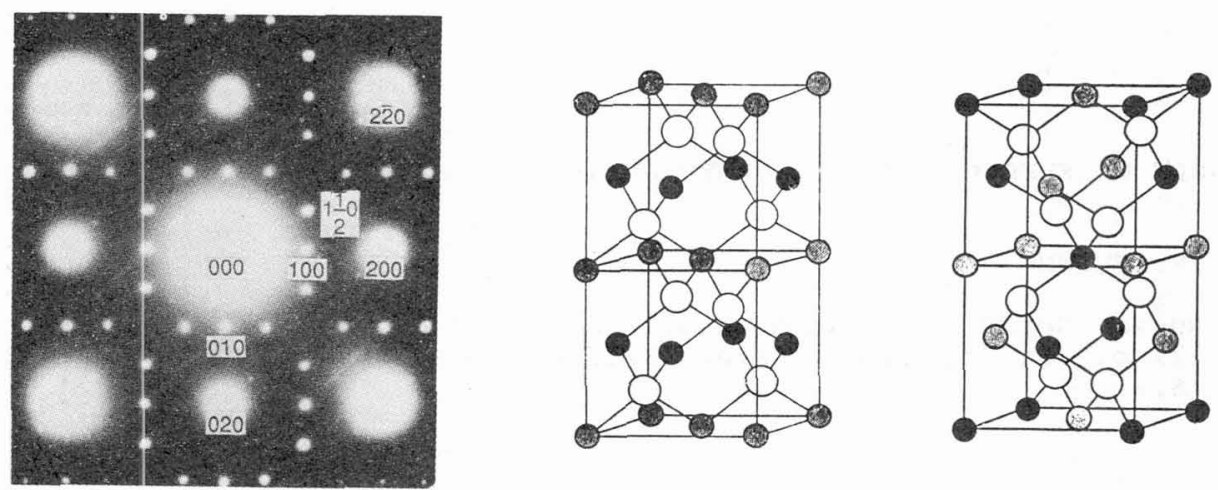

Figure 1. Electron micrograph of epitaxial Ga 2 AsSb grown on [100] InP by MOCVD at $600^{\circ} \mathrm{C}$, showing superlattice lines at $(100)$ and $(11 / 20)$ due respectively to the CuAu and chalcopyrite structures sketched at the right (after H.R. Jen, M.J. Cherng, M.J. Jon and G.B. Stringfellow, Inst. Phys. Conf. Ser. 83, 159 $(1986))$.

In what follows, the formation of strained layers by epitaxial growth is discussed first in Section 2. The relevance of epitaxial strain to suppression of dilute disordered phases is discussed in Section 3 , and the consequent formation of metastable epitaxial compounds (MECs) is explained in Section 4. These phenomena involve delicate energy balances of a few mev/atoms that cannot be treated in a sufficiently accurate way by calculations from first principles. For this reason our discussion takes a different approach.9 The phenomenon is first identified as an instability of the average lattice against the ordering process. The conditions under which ordering takes place are then determined by means of a linear stability analysis. This phenomenology can be fitted to specific systems by choice of parameters; the example of new compounds in mixed salts is discussed in detail. For the present purposes, however, a particular merit of the model is the global view it gives of the phenomenon, and the pitfalls it reveals for efforts to carry through detailed theories for individual systems. Section 5 is a brief sumary.

\section{Strain layers}

Vapor phase growth has the advantage that no bulk phase equilibrates with the growing crystal. Abrupt changes of materials reaching the surface can be effected merely by shuttering arrangements; the absence of inertia permits the growth of finely tailored compositions and almost wholly abrupt heterojunctions. In molecular beam epitaxy (MBE) the atomic species arrive after free flights in molecular beams. Metallorganic chemical vapor deposition employs an inert carrier gas to direct active species to a substrate where they react to produce the desired materials growth. In both cases the substrate temperature and the incident fluxes can be adjusted for optimal growth of high quality single crystal material. MBE grown metals are believed 10 to follow ledge growth mechanisms in which atoms that fall on ledges have time to diffuse to steps before becoming immobile. There results a characteristic linear relationship between optimum growth temperature $T_{g}$ and melting temperature $T_{m}$ (Fig. 2). Somewhat larger relative growth temperatures are required for covalent and polar solids. The semiconductors have smaller intrinsic diffusion and are often grown such that clustering occurs on steps, as evidenced by RHEED oscillations.11 Very little is known about ionic systems. The results in Fig. 2 are unpublished research from the author's laboratory.

There is a strong tendency for chemisorbed atoms to lock in atomic registry with the substrate plane to which they adhere (Fig. 3a). Within fairly wide tolerances this affords the best bonding regardless of the fact that the adsorbed atoms may not be optimally spaced. Since any substrate strain induced by epilayer misfit can be relieved only by elastic deformations to a depth that is comparable with the 


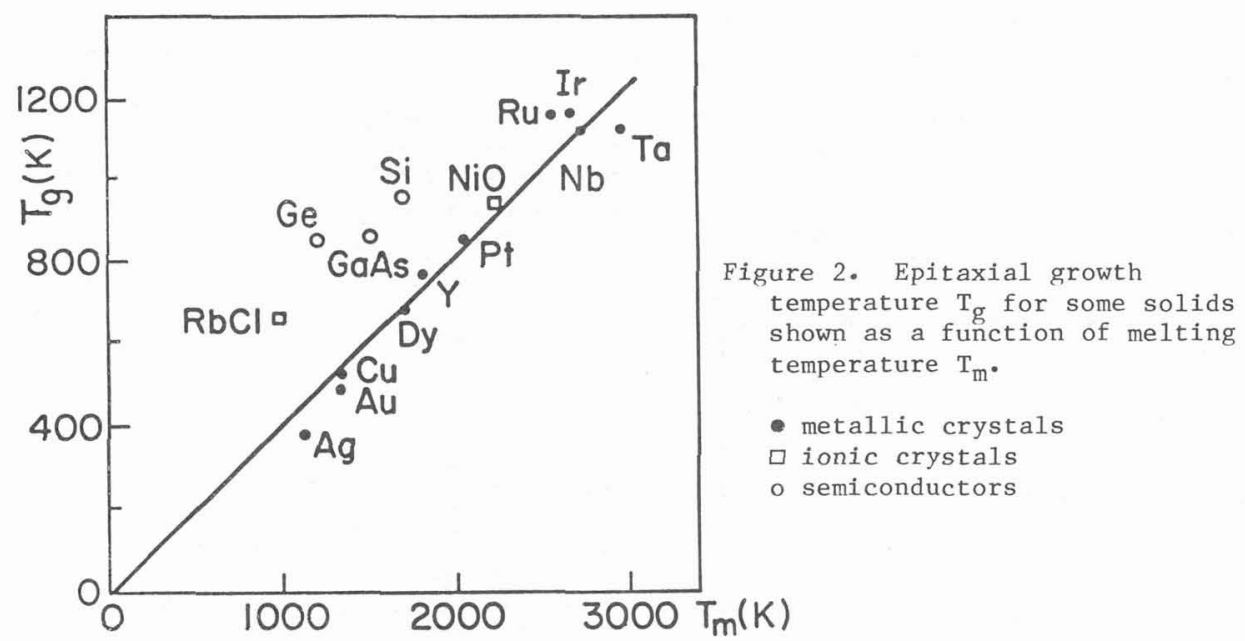

substrate width, it is clear that the energy balance requires essentially all the strain to be in the epilayer and none in the substrate. The same situation recurs layer after layer as atomic planes of a thin epitaxial film are laid down. In this way the initial substrate can provide a self-renewing template by which macroscopic quantities of single crystal material may, in principle, be created in a non-equilibrium configuration. As mentioned above, the actual crystal structure may differ from the equilibrium form. More often the epitaxial film merely exists in a state of homogeneous strain. The complete state of strain is fixed by the constraint of in-plane registry and the fact that the normal stresses are zero. With too great a mismatch, of course, the epitaxial strain must be relieved by defect structures (Fig. 3b) and the resulting imperfect films can grow with a structure close to that of the bulk.: In the presence of a fractional difference $\varepsilon_{0}$ of lattice spacing between the substrate and epilayer the density of interfacial dislocations (Fig. 3b) required to relieve the misfit is proportional to $\varepsilon_{0}$, and so is the required misfit energy.
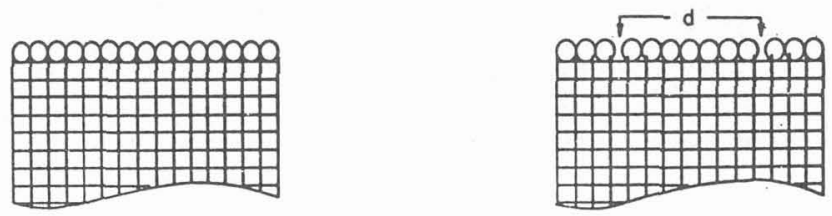

Figure 3. Coherent (left) and defective (right) growth of strained epitaxial films. The coherent film is strained into atomic registry with the substrate lattice parameter, a. For too large a misfit, $\varepsilon_{0}$, defects break the epilayer into domains of width $\mathrm{d} \sim \mathrm{a} / \varepsilon_{0}$.

The energy balance between epitaxial strain and interface defect structure has been analyzed in detail.12,13 Suppose again that the misfit is $\varepsilon_{o}$ of which $\varepsilon$ remains present in the epilayer, thickness $t$, and $\varepsilon_{0}-\varepsilon$ is accommodated by interfacial dislocations. The strain energy is $\sim \beta \varepsilon^{2} t$ (see below) and the interfacial energy $\sim \alpha\left(\varepsilon_{0}-\varepsilon\right)$ with $\alpha$ and $\beta$ materials constants. The fully strained epilayer thus becomes metastable for a critical thickness $t_{0} \geq \beta / \alpha \varepsilon_{0}$, when the energy can be lowered by the generation of misfit dislocations. Thinner films are most stable in the strained form, and this is the basis for stability of important "strain-layer" structures including superlattices with practical application. The occurrence of MECs, also, depends on the fact that epitaxial phases are constrained into atomic registry with the surface. In practice, very perfect films may contain insufficient dislocations to provide strain relief and highly metastable configurations can result. By including thermally activated dislocation processes in an analysis of the stability 
boundary Dodson ${ }^{13}$ reproduces quite well the observed variation of critical thickness $t_{0}$ with misfit $\varepsilon_{0}$, as shown for $S i_{1-x} \mathrm{Ge}_{x}$ on $S i$ in Figure 4. The results involve detailed atomic modeling of dislocation energies to obtain $\alpha$, and an appropriate film strain energy for $B$ which is outlined below. Note that the data include values of $t_{0}$ in the range $10^{3}-10^{4} \AA$. The results revea1 that a good semiquantitative basis exists for understanding strain stability in thick epilayers. By high temperature electron microscopy, Narayan 14 and coworkers observe stress relief at elevated temperatures by direct generation of dislocations at the free surface.

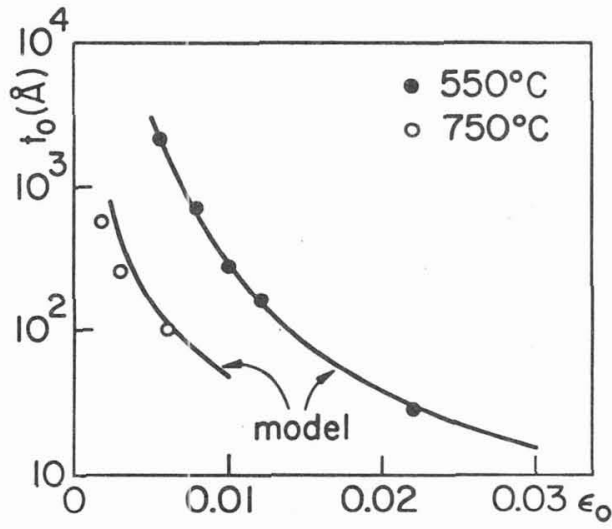

Figure 4. Critical thickness $t_{0}$ of epitaxial films shown as a function of misfit, $\varepsilon_{0}$, at which strain relief by plastic flow becomes perceptible under growth conditions at the temperatures shown (after Dodson and Tsao ${ }^{13}$ ).

It will be convenient for what follows to have explicit forms for the strain free energies of epitaxial films. For crystals many layers thick these are macroscopic considerations defined by the film elastic constants $C_{i j}$ and the epitaxial strain constraint, together with the fact that the normal stresses must be zero. Consider for example a cubic solid grown on its [100] face with in-plane strain $\varepsilon$, and consequent stresses $\sigma$, and with strain $\varepsilon^{\prime}$ and stress zero perpendicular to the film. The relevant stress-strain equation are

$$
\begin{aligned}
& \sigma=C_{11} \varepsilon+C_{12} \varepsilon+C_{12} \varepsilon^{\prime}, \\
& o=2 C_{12} \varepsilon+C_{11} \varepsilon^{\prime} .
\end{aligned}
$$

On solving for $\varepsilon^{\prime}$ the strain free energy for epilayer volume $V$ follows as

$$
F_{e}=V \sum_{i} \sigma_{i} \varepsilon_{i} / 2=\beta V \delta^{2}\left(1-C_{12} / C_{11}\right) / 3,
$$

in which $\delta=3 \varepsilon$ is the volume misfit and $\beta=\left(C_{11}+2 C_{12}\right) / 3$ is the bulk modulus. $F_{e}$ is orientation dependent. In Section 3 we require values of the free energy $F=F_{0}+F_{e}$ with $F_{o}=-B V \delta^{2} / 2$, which defines $J=1-2 F_{e} / B V^{2}$ in the equation

$$
F=-J B V \delta^{2} / 2 \text {. }
$$

From evaluations of $\mathrm{F}_{\mathrm{e}}$ for isotropically strained films in the various orientations one then finds

$$
\begin{array}{ll}
{[100]:} & J=\beta / C_{11} \\
{[111]:} & J=\beta /\left(\beta+4 C_{44} / 3\right) \\
{[110]:} & J=\beta /\left(C_{44}+\left(C_{11}+C_{12}\right) / 2\right) .
\end{array}
$$

These results are employed below in connection with the orientation dependence of MEC formation. 


\section{Metastable epitaxial phases}

Our purpose now is, first, to explain why new metastable phases occur during epitaxial growth and, second, to put forward a simple model that illustrates the behavior. Particular compounds are discussed in Section 4.

An essential ingredient is that the material contain two competing species that exhibit similar but not identical properties, so that they are not fully interchangeable. If large enough, this difference can prevent substantial miscibility in the bulk, with the concentrated bulk mixture decomposing into two dilute solutions. Under certain circumstances this decomposition-can be prevented in an epitaxial film, so that the concentrated mixture is preferred instead. Any ordered compound can then form provided only that it is more stable than the concentrated solution, whereas in the bulk the dilute phases occur in preference to both the compound and the concentrated solution. This suppression of the two dilute phases can be engineered by making both of them misfit an epitaxial constraint for which the compound has little or no misfit. For this mechanism to operate the free energies of the various disordered and ordered phases must take the forms shown in Fig. 5. In the disordered bulk phase at $0 \mathrm{~K}$ the free energy bows upward as a function of composition $x$ because of the atomic misfit. In the epitaxial phase, however, the free energy of the dilute phases is increased (arrows) by epitaxial misfit to leave the concentrated phase favored instead. Any compound that lies lower than the disordered phase (broken lines) may then form.

Figure 5. Free energy as a function of composition $x$ for bulk (left) and epitaxial (right) disordered $A_{x} B_{1-x}$ (thick curves) and ordered $A B$ (broken lines). 9 Faint lines include mixing entropy for $T \neq 0$.

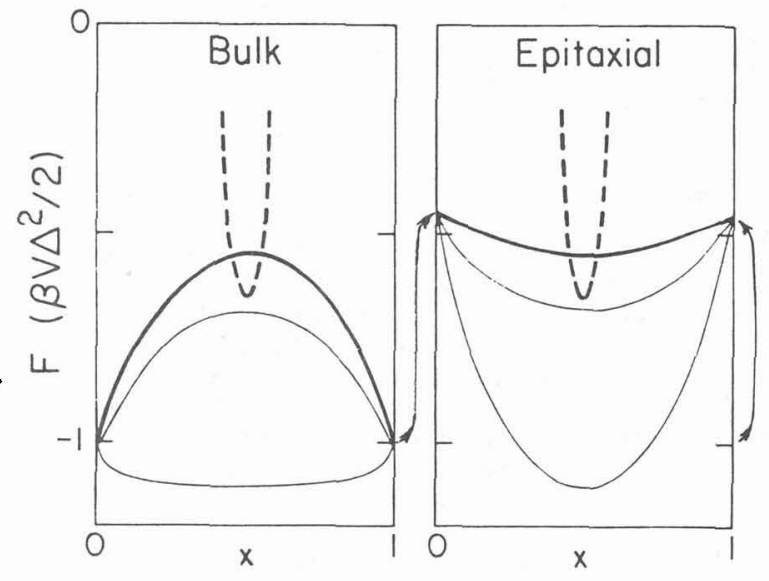

Using detailed electronic calculations Martins and Zucker 15 have studied the total energy of $\mathrm{Si}$, Ge and two ordered phases of SiGe on Si substrates, with the lattices relaxed perpendicular to the interface. They find that rhombohedral (R) Sige is preferred over the zincblende structure ( $Z$ ) and that epitaxial (R) on $S i$ has a lower total energy than that of the equivalent quantities of $\mathrm{Si}$ and Ge separately on Si. A tendency for (R) Sige to form is thus indicated, in accord with experiments. 4 In other research Zucker and coworker ${ }^{16}$ examine a variety of $A_{4} B_{n} C_{4-n}$ compounds in an effort to understand the way energetics determines both short range and long range order in these systems. The most favored structures are those that best accommodate the atomic differences in a strain-free manner. It should be mentioned in connection with these pioneering efforts that as the relevant energy differences may amount only to a few meV per atom the entire problem is an extreme challenge for calculations from first principles. In addition, of course, it is the free energy, not the potential energy alone, that determines phase equilibrium. Just one aspect of this latter difficulty is that the vibrational free energy of either competing phase at $\mathrm{T}_{\mathrm{g}}$ exceeds the calculated energy differences by about two order of magnitude. Such terms must eventually be included in calculations of the resulting phase equilibrium. 
In place of detailed electronic calculations we describe here a phenomenological theory, represented by a model Hamiltonian, that allows the main model effects to be derived quantitatively in a simple way. 9 The desired phase behavior follows from a linear stability analysis of an average lattice on whose properties the atomic difference driving phase change form a precisely defined perturbation. The average lattice has the Hamiltonian

$$
H=-\sum_{s}\left(\hbar^{2} / 2 M_{s}\right) \nabla_{s}^{2}+\frac{1}{2} \vec{x} \cdot \ddot{c} \cdot \vec{x},
$$

in which $\ddot{C}$ is the force constant matrix and $\vec{x}$ the vector of atomic displacements; the index s runs over all three coordinates of all atoms. We employ as the atomic perturbation

$$
H^{*}=\sum_{i}\left[E_{i}^{ \pm} \pm \lambda \sum_{j} x_{i j}\right]
$$

in which the atoms with the active differences, indexed by $i$, are linearly coupled to other atoms $j$ of the material. In practice we confine attention here to near neighbor coupling only, through the changes of bond lengths $x_{i j}$. The i-specific energies $E_{i}$ do not enter into phase equilibration and may be dropped.

We point. out three helpful features of the chosen Hamiltonian. First, when all $\mathrm{x}_{i j}=0, H^{\prime}=0$ for all permutations of the active atoms among their accessible sites. Thus, the relative potential energies of the different phases are just their relaxation energies, from the related configurations with $x_{i j}=0$, under the driving force of $H^{\prime}$. Second, the change of vibxational free energy between different configurations at high temperatures can be calculated exactly and is zero. This follows from the fact that the force constant matrix is not modified by interchanges of $i$ atoms or by relaxation, owing to the linear coupling. The lattice eigenmodes and their frequencies $\omega_{\text {, and }} \omega_{\text {i }}$ all change between the two configurations, but contribute to the consequent absence of free energy change only through $\delta F_{\mathrm{V}}=-\mathrm{kT} \sum_{i} \ln \left(\omega_{i}^{\prime} / \omega_{i}\right) \equiv 0$, in which the last step follows from a well known product rule on the eigenvalues.17 Finally, the coupling coefficient $\lambda$ is not free but instead is determined by the atomic misfit being modeled. Here we study only volume (bond length) misfit. The summed lattice volume energy $\langle H\rangle=B(\delta V)^{2} / 2 \mathrm{~V}$ and misfit energy $\left\langle H^{\prime}\right\rangle=-\mathrm{nN} \lambda \mathrm{a}(\delta \mathrm{V} / 3 \mathrm{~V})$ are minimized for $\delta \mathrm{V}=\mathrm{V} \Delta$ only when $\lambda=3 \beta \Omega \Delta \mathrm{an}$, with $\mathrm{F}_{\mathrm{r}}=$ $-\beta V \Delta^{2} / 2$ the relaxation free energy. Here $\beta$ is the bulk modulus, and $\Omega=V / N$ is the volume per $i$ atom, with $N$ in the total volume $v$, each having $n$ near neighbor bonds of length a. With this selection of $\lambda$ the Hamiltonian is completely fixed by the fractional volume change $2 \Delta$ between lattices containing wholly + species or wholly - species of $i$ atoms.

Because vibrational free energy thus causes no driving force for phase change, the phenomenon of metastable phase formation for the present harmonic model arises entirely from the potential energy of the static lattice. As in all linear coupling cases 17 , the energy lowering by relaxation from the state $x_{i j}=0$ is

$$
F=-\sum_{i} w_{i}^{2} \xi_{i}^{2} / 2
$$

in which the $\omega_{i}$ are phonon frequencies and the $\xi_{i}$ the equilibrium normal mode amplitudes driven by $\lambda \neq 0$. Thus, the stability question is resolved by computing the $\left\langle\xi_{i}{ }^{2}\right\rangle$ for the relevant ordered compounds and disordered phases. As the ordered structures are specific to particular cases we defer their discussion to the example in Section 4. For the present much can be learned separately about the disordered phase and epitaxy, so it is to a discussion of these topics that we now turn. 
It is simplest to consider the relaxation free energy of the disordered solid from the state $x_{i j}=0(\operatorname{all} i, j)$ as a sequence of two steps. First the 1 atoms for $x=0$ are rearranged so that the two active species occupy two separate regions of the solid, which are then allowed to relax independently. In this relaxation the larger species cause their solid to change volume by $v_{1} / 2 \rightarrow(1+\Delta) v_{1} / 2$ and the smaller species a change $V_{2} / 2 \rightarrow(1-\Delta) V_{2} / 2$. The total free energy change is $-\beta V \Delta^{2} / 2$ with $V=$ $\mathrm{v}_{1}+\mathrm{v}_{2}$. The second step of our process is to take the two resulting solids and mix them to form the relaxed disordered solid. This is a classical problem of metallurgy and ceramics whose resolution is that the free energy change as a function of composition $x$ is about $180.9 B V \Delta^{2} x(1-x)$, which yields a net relaxation energy for the bulk material of

$$
\mathrm{F}_{\mathrm{r}}=-\frac{1}{2} \beta V \Delta^{2}[1-1.8 \mathrm{x}(1-\mathrm{x})]
$$

To be consistent, the change of lattice volume varies with $x$ as $\delta(x)=\Delta(2 x-1)$.

For an epitaxial film, the epitaxial strain energy (Section 2) must be added to the bulk free energy to obtain the net free energy. Suppose that the substrate offers a lattice match to the composition $x_{0}$, so that the actual compound of composition $x$ misfits by a strain $2\left(x_{0}-x\right) \Delta$ (note that the change is $2 \Delta$ from $x_{0}=0$ to $x_{0}=1$ ). The free energy of the film for [100] growth is, (cf Section 2):

$$
F_{e}=-\frac{1}{2} \beta V \Delta^{2}\left[1-1.8 x(1-x)-8\left(x-x_{o}\right)^{2}\left(1-C_{12} / C_{11}\right) / 3\right]
$$

One finds $\partial^{2} F / \partial x^{2}=\left(\beta V \Delta^{2} / 2\right)(4 \cdot 4-8 J)$ so that the epitaxial system is stable against decomposition into dilute phases whenever $J<0.55$. This result holds for all orientations of the growth plane, given the correct $J$ for that plane. It holds for all choices of substrate lattice spacing (i.e. all $\mathrm{x}_{\mathrm{o}}$ ). Since the mixing entropy further stabilizes all compositions the result $\mathrm{J}<0.55$ is also sufficient at all temperatures. Results analogous to these have been described earlier by Stringfellow 19 and by de Cremoux 20 to explain why semiconductor alloy films can be prepared epitaxially from components that are immiscible at the same composition of the bulk.

From a more fundamental perspective we note that the stability holds for all fourier components of composition and, indeed, is likely to be augmented by insipient surface energy 21 terms. Any phase coincidence among fourier components that tends to nucleate incoherent boundaries is subject to energetic constraints of the type discussed in Section 2. In the long run these questions of bulk epitaxial stability may be made moot by the likelihood that only the outer layer of atoms remains mobile at the growth temperature. The consequences of this kinetic factor, and their effect on fluctuation growth from one layer to the next, remain to be elucidated.

Table 1. Anisotropy of epitaxial strain free energy for several types of materials, as calculated from the coefficient J. (see equs 7 ). J $<0.55$ indicates stability of the disordered phase against decomposition into dilute components at $\mathrm{T}=0$. The most stabilized orientations are boxed.

\begin{tabular}{l|lll}
\hline & {$[100]$} & {$[110]$} & {$[111]$} \\
\hline $\mathrm{Si}$ & 0.590 & 0.503 & 0.480 \\
$\mathrm{Ge}$ & 0.584 & 0.480 & 0.453 \\
$\mathrm{GaAs}$ & 0.638 & 0.531 & 0.494 \\
$\mathrm{InSb}$ & 0.697 & 0.570 & 0.538 \\
$\mathrm{Mg0}$ & 0.536 & 0.458 & 0.437 \\
$\mathrm{NaC1}$ & 0.503 & 0.568 & 0.593 \\
$\mathrm{RbC1}$ & 0.422 & 0.612 & 0.720 \\
$\mathrm{CsBr}$ & 0.507 & 0.574 & 0.600 \\
$\mathrm{CsI}$ & 0.515 & 0.580 & 0.605 \\
\hline
\end{tabular}


The predictions these results yield for specific materials are of some interest. Table 1 surveys values of $\mathrm{J}$ for various solids calculated using elastic constants tabulated by Kittel.22 For each material at least one orientation has $\mathrm{J}<0.55$, indicating a net strain energy that stabilizes the concentrated disordered phase in the epilayer (see Fig. 5b). For semiconductors [111] is preferred and for alkali halides [100]. Most notable is the fine balance of the energetics revealed by the results. In no case does J differ from 0.55 by more than about 0.15 , just $15 \%$ of the segregation energy $\beta V \Delta^{2} / 2$. Given added mixing entropy for growth at $\mathrm{T}_{\mathrm{g}} \sim 10^{3} \mathrm{~K}$, rather widespread epitaxial solubility is expected. The occurrence of ordered phases (MECs) depends separately on the energetics of compound formation. This is discussed in the next section.

\section{New compounds of alkali halides}

The formation of new ordered compounds is driven by the accessibility of epitaxial ordered phases that have lower free energy than the epitaxial disordered phase discussed above (Section 3). To analyze the phenomenon we must therefore determine the free energy released by compound formation. For this purpose we need merely calculate the energy change as the active species drives relaxation from the standard state $x_{i j}=0$. It is most useful to carry through a particular model in detail. For this reason we continue here with the linear stability treatment of volume misfit, and the compound formation to which it gives rise. Only in ionic and molecular crystals does the notion of atomic size play a well defined role. The discussion that follows therefore focuses largely to the case of alkali halides, for which realistic predictions can be made. Only for the enormous misfit $\Delta=0.54$ of CsF-LiF has evidence for ordered compound formation of a bulk mixed alkali halide received experimental support ${ }^{23}$. For conciseness, only the formation of compounds with equal numbers of the two active species is considered although, as indicated above, the same formalism may be applied to compound having other compositions, $x_{0}$, by selecting a substrate that lattice matches this composition.

Figure 6. Model ionic lattice including small and large (circled) cations.9 Growth planes are stacked with successive displacements $(a, 0)$. Arrows on one diagonal indicate inplane anion phonon displacements at L (see text).

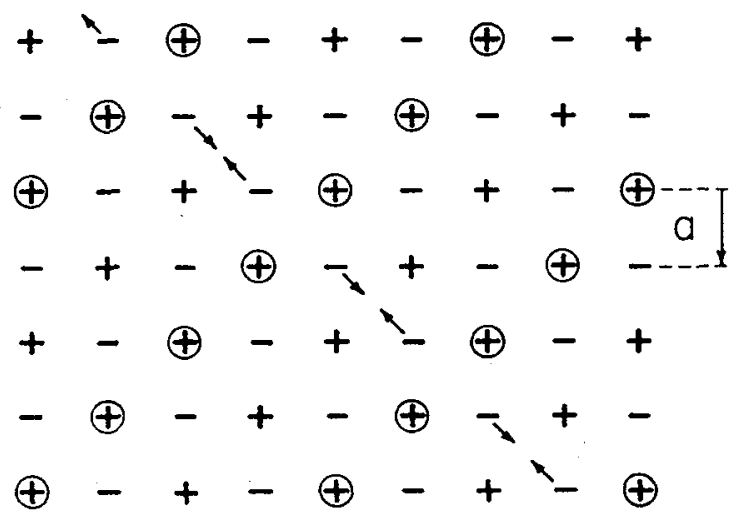

A model rocksalt-derived compound having two different cations in equal proportions is shown as an example in Fig. 6. Successive planes are stacked with displacements of $a$, so that the anions and cation species form [111] sheets. As indicated, the size difference between active cations drives anion relaxation in the pattern of the longitudinal phonon at the $L$ point of the reduced Brillouin Zone, i.e. (111). From the force $\lambda x$ and the phonon potential energy $\omega_{L}^{2} \xi_{L}^{2} / 2$ one calculates an energy change from compound formation 


$$
F_{c}=-\frac{1}{2} \beta V \Delta^{2} K
$$

with $\mathrm{K}=6 \mathrm{Ba} / \mathrm{M \omega} \mathrm{L}_{\mathrm{L}}^{2}$ for the compound shown. Very similar results are found in other cases.* Thus, both for the CsC1 structure with the active cations separated in [100] sheets, and for the CuAu structure of $\mathrm{Ga}_{2} \mathrm{AsSb}$ (see above), $\mathrm{K}=4 \mathrm{Ba} / \mathrm{Mw} \mathrm{X}_{\mathrm{X}}$, with $\mathrm{X}$ the zone boundary at (100).

Table 2. Values of constants $J=\beta / C_{11}$ (upper) and $\mathrm{K}=6 \beta \mathrm{Ba} / \mathrm{M \omega}_{\mathrm{L}}^{2}$ (1ower) calculated from data for fcc alkali halides (see text). With $K>J$ the epitaxial ordered compound at low temperature is stable against sizeinduced decomposition into disordered epitaxial binaries for all lattices except LiF. Values of $\mathrm{Mw}_{\mathrm{L}}^{2}$ generally differed little for anions and cations; the average is used in the table.

\begin{tabular}{|c|c|c|c|c|c|}
\hline & Li & $\mathrm{Na}$ & K & $\mathrm{Rb}$ & $\mathrm{Cs}$ \\
\hline $\mathbf{F}$ & $\begin{array}{l}0.586 \\
0.49\end{array}$ & $\begin{array}{l}0.497 \\
0.58\end{array}$ & $\begin{array}{l}0.484 \\
0.58\end{array}$ & $\begin{array}{l}0.480 \\
0.61\end{array}$ & $\begin{array}{l}0.415 \\
0.67\end{array}$ \\
\hline $\mathrm{Cl}$ & $\begin{array}{l}0.638 \\
. \quad .\end{array}$ & $\begin{array}{l}0.437 \\
0.61\end{array}$ & $\begin{array}{l}0.437 \\
0.61\end{array}$ & $\begin{array}{l}0.445 \\
0.62\end{array}$ & \\
\hline $\mathrm{Br}$ & $\begin{array}{l}0.651 \\
. .\end{array}$ & $\begin{array}{l}0.438 \\
0.63\end{array}$ & $\begin{array}{l}0.438 \\
0.63\end{array}$ & $\begin{array}{l}0.434 \\
0.60\end{array}$ & \\
\hline I & $\begin{array}{l}0.660 \\
.\end{array}$ & $\begin{array}{l}0.447 \\
0.61\end{array}$ & $\begin{array}{l}0.447 \\
0.61\end{array}$ & $\begin{array}{l}0.430 \\
0.56\end{array}$ & \\
\hline
\end{tabular}

To provide a broad picture of a particular example Table 2 compares values of $\mathrm{J}$ and $\mathrm{K}$ for the rocksalt compound shown above using experimental phonon frequencies 24 and elastic constants of alkali halide lattices.25 For all cases except LiF we find $\mathrm{J}<0.55<\mathrm{K}$, showing that the lattice free energy of the epitaxial compound generally lies lowest, and those of the concentrated epitaxial and segregated epitaxial phases progressively higher. A remarkable consistency of the linear stability criteria from one lattice to the next is evident, regardless of the narrow margin of the energy balance. Note that the mixing entropy of the disordered phase at the growth temperature is neglected in this comparison, and must be included in specific predictions. With $\mathrm{T}_{\mathrm{g}} \sim 500^{\circ} \mathrm{C}$, for example, this amounts to $\sim 50 \mathrm{meV} / \mathrm{active}$ atom. A bulk misfit energy $\beta V \Delta^{2} / 2 \sim 0.5 \mathrm{eV} /$ active atom is therefore needed before the epitaxial process can clearly favor compound formation. Since, invariably, $\mathrm{T}_{\mathrm{g}} \sim 0.5$ $\mathrm{T}_{\mathrm{m}}$ (Fig. 1) one sees that new epitaxial phases can form by the mechanisms discussed here only for systems with very limited bulk solid solubility.

* Note in passing that similar results apply to the disordered alloy, with all fourier components of density relaxing under random driving force amplitudes. The $\omega^{-2}$ dependence explains why equations involving only the long wavelength 1imit (i.e. elastic constants) provide a useful description. 
There is a valuable extension of the preceeding results (which are derived for a harmonic lattice) to systems that are more realistically anharmonic. A clearer picture of the difficulties faced by accurate calculations is thereby obtained. The self consistent phonon approximation allows any particular lattice to be regarded as having a potential energy, $U_{o}$, and harmonic phonon frequencies, $\omega$, (including elastic constants) that are, however, volume dependent and, in addition, mildly temperature dependent. It: is thus useful to recognize that the linear stability analysis presented above remains valid in this approximation provided that the actual potentials and frequencies relevant to the particular state of the lattice are employed in the equations presented above. The consequences for phase stability may be quite striking. Fig. 7 shows by way of example the observed dielectric constant and optic mode frequencies as a function of $T / T_{m}$ in some salts.26 It turns out that the fractional frequency changes are large and vary from one phonon to the next, the optic moge effects being among the largest. Consequently it is possible for ratios like $\beta / \omega_{\mathrm{L}}$ that define $K$ to vary considerably with $T$. Since the margin of compound stability over the disordered phase depends on differences $\sim 10 \%$ of $\mathrm{K}$ (Table 2 ) one is drawn to the conclusion that anharmonic phenomena of this type can possibly play a central role in determining what phase structures appear in real cases. No efforts to date have been made to refine the theory with sufficient accuracy to illuminate the way new epitaxial compounds are formed in practical cases.

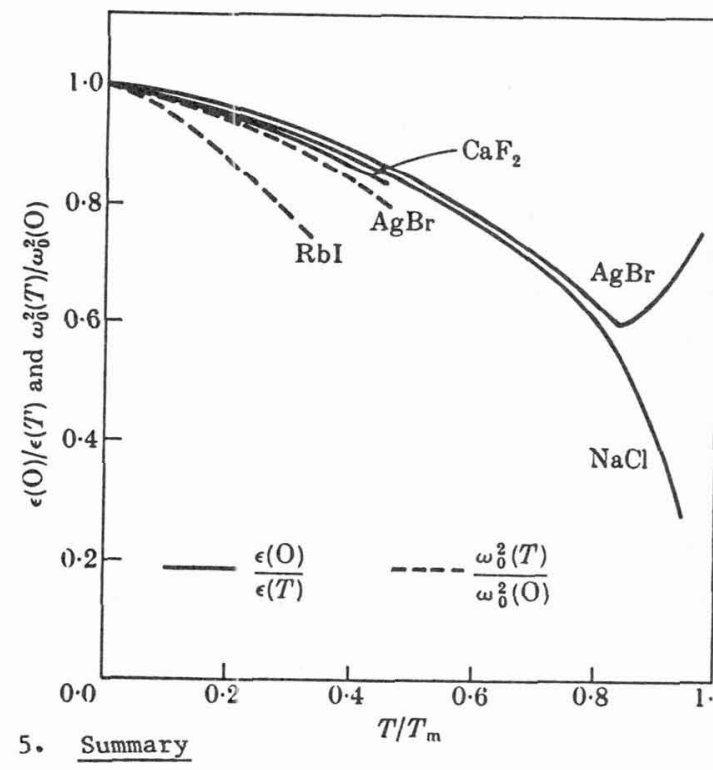

Figure 7. Temperature dependence of the inverse dielectric constant (at $\sim 10^{10} \mathrm{~Hz}$ ). The optic mode frequency is more strongly temperature dependent as shown by the data (broken lines). 26

The preceeding pages describe how new ordered metastable phases may occur pseudomorphically during epitaxial growth. For the mechanism described here the strain of epitaxial misfit is an essential ingredient needed to inhibit segregation of the material into dilute disordered phases. The theoretical stability limits of such epitaxial films against strain-relief by plastic flow have been reviewed and pertinent experiments described. The strain permits ordered compounds to compete with the concentrated disordered phase and so to form whenever their ordering energy exceeds the opposing entropy of mixing.- A linear stability analysis has been used to describe the relevant free energy differences for the particular example of volume misfit between the active lons. The analysis has been carried through in detail for mixed alkali halides, a case in which the volume misfit is quite well defined. It appears very probable that mixed alkali halide compounds should occur during epitaxial growth, whereas in the bulk they are not usually observed.

Several general conclusions emerge from the linear stability analysis. It turns out that the free energy balances are quite delicate as they involve differences an order of magnitude smaller than the total interaction energy of the active species. Almost all the lattices studied possess at least one orientation for which segregation into dilute phases is suppressed, the face-dependence arising from elastic anisotropy alone. The [100] face is generally favored for alkali halides and [lll] for semiconductors. Direct calculations for chosen ordered compounds using 
measured phonon frequencies show that the disordered lattices at low $T$ are indeed unstable against ordering. At the growth temperature the mixing entropy must be included but it seems likely that this may be overwhelmed by anharmonic effects which, while analyzed, remain to be made quantitative. Additional complications due to surface kinetics and to a multiplicity of equivalent growth orientations are likely to remain present in experiments for some time to come.

A strength of the present analysis is that the energetics are described entirely in terms of the interaction free energy of the competing ions and vibrational properties of the basic lattice. The energy differences therefore remain significant fractions $(\sim 10 \%)$ of the total energy treated. This allows the investigation of systematics, as in the orientation dependences (Table 1) or the broad validity of $\mathrm{J}<0.55<\mathrm{K}$ for simple salts (Table 2). It also permits the analysis of complexities such as the anharmonic effect on energies of compounds. The particular formulation given here is limited to volume dependent energies. Experimental evidence for such case as $A g A u$ and $\mathrm{Ga}_{\mathrm{X}} \mathrm{Al}_{1-\mathrm{x}} \mathrm{As}$, for which the volume effects are minimal, indicates that other processes also must contribute. The ideas of Zunger and coworkers are relevant here.16 It would be valuable for the future to identify other complementary degrees of internal freedom to which simflar components of a more general stability analysis could be applied.

The participation of the author in this conference was supported through the University of Illinois Materials Research Laboratory by NSF grant DMR-86-12860. Thanks are expressed to Drs. B.W. Dodson, G.B. Stringfellow and A. Zunger for providing manuscripts that are currently in press.

\section{References}

1. For early references see W.A. Jesser, Mater. Sci. Eng. 4, 279 (1969).

2. See e.g., G.A. Prinz, Phys. Rev. Lett.54, 1051 (1985); M. Onellion, M.A. Thomson, J.L. Erskine, C.B. Duke and A. Paton, Surf. Sci. 179. 219 (1987).

3. R.F.C. Farrow, J. Vac. Sci. Technol. B1, 222 (1983).

4. A. Ourmazd and J.C. Bean, Phys. Rev. Lett. 55, 765 (1985).

5. T.S. Kuan, T.F. Kuech, W.I. Wang and E.L. Wilkie, Phys. Rev. Lett. 54, 210 (1985).

6. H.R. Jen, Y.T. Cherng and G.B. Stringfellow, Appl. Phys. Lett. 48, 1603 (1986).

7. H.R. Jen, M.J. Jon, Y.T. Cherng and G.B. Stringfellow (to be published).

8. H.E. Cook, Thesis, Northwestern University (1966, unpub1ished).

9. C.P. Flynn, Phys. Rev. Lett. 57, 599 (1986).

10. J.E. Cunningham, J.A. Dura and C.P. Flynn, Trans. AIME (in press).

11. J.H. Neave, B.A. Joyce, P.J. Dobson and N.Norton, J. Appl. Phys. A31, 1 (1983); J.H. Neave, P.J. Dobson, B.A. Joyce and J. Zhang, Appl. Phys. Lett. 47, 100 (1985).

12. J.W. Matthews and A.E. Blakeslee, J. Cryst. Growth 27, 118 (1974).

13. B.W. Dodson and P.A. Taylor, Appl. Phys. Lett. 49, 642 (1986); B.W. Dodson and J.Y. Tsao, App1. Phys. Lett (in press).

14. See, e.g., the contributions of J. Narayan to this symposium.

15. J.L. Martins and A. Zunger, Phys. Rev. Lett. 56, 1400 (1986).

16. See, e.g., A.A. Mbaye, A. Zunger and D.M. Wood, Appl. Phys. Lett. 49, 782 (1986).

17. See, e.g., C.P. Flynn, Point Defects and Diffusion (Oxford, 1972).

18. See, e.g., J. Friedel, Adv. Phys. 3, 446 (1954); in the present work this formula has ben found broadly satisfactory also for the alkali halide results of D.L. Fancher and G.R. Barsch, J. Phys. Chem. Solids 30, 2503 (1969).

19. G.B. Stringfellow, J. Crystal Growth 27, 21 (1974); ibid, 58, 1914 (1982).

20. B. de Cremoux, J. Phys. 43, Suppl. 12, C5, 19 (1982).

21. H.E. Cook and J.E. Hilliard, Appl. Phys. Lett. 8, 74 (1966).

22. C. Kittel, Introduction to Solid State Physics (Wiley, 1971).

23. G.A. Bukhalova and D.V. Sementsova, Russ. J. Inorg. Chem. 10, 1025 (1965).

24. H. Bilz and W. Kress, Phonon Dispersion Curves in Insulators (Springer-Verlag, Berlin 1979).

25. G. Simmons and H. Wang, Single Crystal Elastic Constants (MIT Press, Cambridge, 1971 ).

26. See ref. 17, p 599. 